\title{
Reduction and Carburization of Iron Oxides for Fischer-Tropsch Synthesis
}

Monia Nielsen ${ }^{1}$, Asger Moss ${ }^{1}$, Anton Simon Bjørnlund ${ }^{1}$, Xi Liu ${ }^{2}$, Axel Knop-Gericke ${ }^{3}$, Alexander Yu. Klyushin $^{3}$, Jan-Dierk Grunwaldt ${ }^{4}$, Thomas Sheppard ${ }^{4}$, Dmitry E. Doronkin ${ }^{4}$, Anna Zimina ${ }^{4}$, Thomas Erik Lyck Smithuysen ${ }^{1}$, Yakub Fam ${ }^{1}$, Christian Danvad Damsgaard ${ }^{1}$, Jakob Wagner ${ }^{5}$ and Thomas Hansen ${ }^{1}$

${ }^{1}$ Technical University of Denmark, Kgs. Lyngby, Hovedstaden, Denmark, ${ }^{2}$ Shanghai Jiao Tong University, Shanghai, Shanghai, China (People's Republic), ${ }^{3}$ Fritz Haber Institute of the Max Planck Society, Berlin, Berlin, Germany, ${ }^{4}$ Karlsruhe Institute of Technology, Karlsruhe, Baden-Wurttemberg, Germany, ${ }^{5}$ DTU Nanolab, Technical University of Denmark, Kgs. Lyngby, Hovedstaden, Denmark

Fischer-Tropsch Synthesis (FTS) is the chemical reaction in which a mixture of carbon monoxide and hydrogen (syngas) is converted into mainly hydrocarbons which are subsequently used for making liquid fuels and chemicals. Iron and cobalt are the most commonly used catalysts in industrial FTS [1]. Compared to cobalt, iron is cheaper as it is one of the most abundant elements in the crust of the earth. Iron catalysts are less active but also less impacted by operating conditions, i.e. pressure and temperature.

The activation of an iron-based FTS catalysts typically consists of two steps: reduction in hydrogen followed by carburization in either $\mathrm{CO}$ or syngas mixture. Alternatively, both steps can be carried out simultaneously in syngas. A combination of different complementary in situ techniques was used to gain insight into the behavior of Fe-based FTS catalysts during these steps. In situ XRD was used to identify the crystalline phases present during both steps. From the XRD data, an increase in reduction rate was observed with increasing temperature. In situ X-ray diffraction experiments in $\mathrm{H}_{2}$ at a flow of $2 \mathrm{Nml} / \mathrm{min}$ corresponding to a $\mathrm{H}_{2}$ partial pressure of 14 mbar at the sample show the temperature dependence of the reduction process. Metallic iron is present already at around $400^{\circ} \mathrm{C}$ and at $700^{\circ} \mathrm{C}$ the sample is fully reduced. However, these experiments do not provide any information on how the reduction properties depends on the structure.

To further investigate the structural effects of the reduction process, environmental transmission electron microscopy (ETEM) was used [2]. The experiments were carried out at low pressure using an aberration corrected FEI Titan 80-300 E-cell, operated at $300 \mathrm{kV}$. The sample was dispersed in ethanol and sonicated for $15 \mathrm{~min}$. prior to drop casting onto a through-hole Wildfire heating chip from DENSsolutions. Iron oxide was reduced in situ in hydrogen at temperatures up to $500^{\circ} \mathrm{C}$ at various pressures in the range 1.3 3.8 mbar. High-pressure studies were carried out using the Climate closed gas-cell holder from DENSsolutions, with a gas flow up to $0.9 \mathrm{Nml} / \mathrm{min}$ and a maximum pressure of $1 \mathrm{~atm}$. In order to minimize the effect of the electron beam, the beam was turned off while ramping the temperature between each image/diffraction/spectrum acquisition.

Both oxides and metallic iron were observed during the reduction using in situ XRD. These were further confirmed when reducing iron oxide in the ETEM. With a pressure of $1.3 \mathrm{mbar} \mathrm{H}_{2}$ at a flow of $2 \mathrm{Nml} / \mathrm{min}$, iron oxide was reduced to metallic iron at $500^{\circ} \mathrm{C}$ as confirmed by electron energy-loss spectroscopy (EELS), Figure 1. With limited beam influence, hematite was completely reduced at $500^{\circ} \mathrm{C}$. At $450^{\circ} \mathrm{C}$, imaging and diffraction indicated a clear dependence on agglomerate size, Figure 2. At this temperature, diffraction patterns from the larger agglomerate in Figure $2 \mathrm{a}$ indicate the presence of both metallic iron as 
well as hematite while under the same conditions, the smaller agglomerate in Figure $2 \mathrm{~b}$ show only metallic iron. A possible explanation could be the presence of water produced during the reduction process.

In conclusion, in the ETEM, complete reduction to metallic was demonstrated and an effect of agglomerate size was apparent. Both ETEM and high pressure experiments indicate that smaller agglomerates reduce at lower temperatures than larger agglomerates.

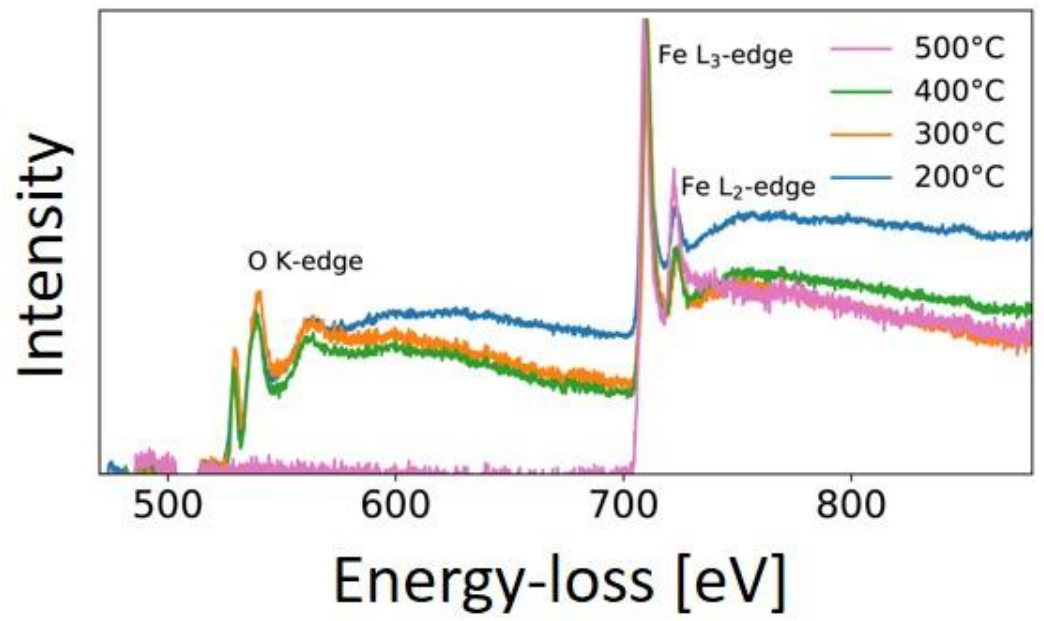

Figure 1. EELS data from the reduction of hematite at increasing temperatures, showing the oxygen Kedge $(\sim 532 \mathrm{eV})$ and the iron L-edge $(\sim 708 \mathrm{eV})$. The data is normalized at the peak intensity at the Fe L3edge.
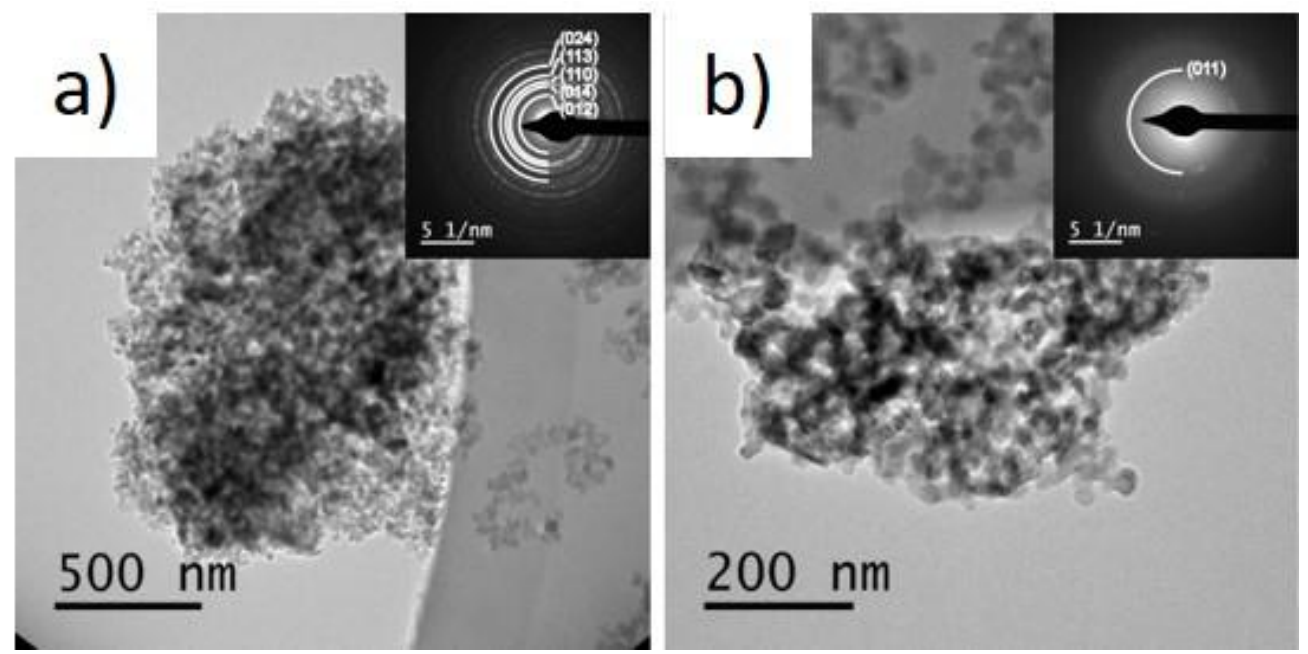

Figure 2. Two agglomerates of different size with corresponding electron diffraction patterns acquired at $450^{\circ} \mathrm{C}$, showing a) both the presence of hematite and b) metallic iron. The images and diffraction patterns were acquired under the same conditions.

\section{References}

[1] B. H. Davis, Catal. Today 84, (2003) 83-98.

[2] T. W. Hansen, J. B. Wagner, R. E. Dunin-Borkowski, Mater. Sci. Technol. 26, (2010) 1338-1344. 